Research Paper

\title{
Treatment of Advanced or Recurrent Cervical Cancer with Cisplatin or Cisplatin Containing Regimens: A Cost Eifective Analysis
}

\author{
John P. Geisler ${ }^{\bowtie}$, Jayanth Swathirajan, Katherine L. Wood, Kelly J. Manahan \\ Division of Gynecologic Oncology, Department of Obstetrics and Gynecology, University of Toledo College of Medicine and \\ Life Sciences, Toledo, Ohio, USA.
}

\begin{abstract}
$\triangle$ Corresponding author: John P. Geisler, MD. University of Toledo College of Medicine and Life Sciences, Division of Gynecologic Oncology, 3120 Glendale Avenue, Mail Stop 1194, Toledo, OH 43614. Email: john.geisler@utoledo.edu.
\end{abstract}

( ) Ivyspring International Publisher. This is an open-access article distributed under the terms of the Creative Commons License (http://creativecommons.org/ licenses/by-nc-nd/3.0/). Reproduction is permitted for personal, noncommercial use, provided that the article is in whole, unmodified, and properly cited.

Received: 2012.07.02; Accepted: 2012.10.17; Published: 2012.11.01

\begin{abstract}
Background: Trials have demonstrated improvements in survival with adding paclitaxel $(\mathrm{P})$ or topotecan $(T)$ to cisplatin $(C)$ for the treatment of advanced cervical cancer. We sought to evaluate the cost effectiveness of these regimens.

Methods: A decision model was developed based on Gynecologic Oncology Group (GOG) protocols 169 and I79. Arm I is 6 cycles of cisplatin. Arm 2 is 6 cycles of CP while arm 3 is 6 cycles of CT. Parameters include overall survival (OS), cost and complications. Sensitivity analyses were performed.

Results: The incremental cost-effectiveness ratio (ICER) for $C$ versus $C P$ is $\$ 13,654$ /quality-adjusted life-year (QALY) gained. For CT compared to C, the ICER is $\$ 152,327 / \mathrm{QALY}$. When compared simultaneously, CT is dominated. At a willingness to pay (WTP) threshold of $\$ 50,000 / Q A L Y, C$ is the preferred option but CP is acceptable. Sensitivity analyses suggest that CT would become the preferred option if it was to improve OS to 24 months (compared to 9.4 months).

Conclusions: In this model, CP is an acceptable alternative to cisplatin for the treatment of these patients with an increase in cost of only \$13,654/QALY. The addition of topotecan did not increase survival enough to justify the increased cost.
\end{abstract}

Key words: cervical cancer, chemotherapy, cisplatin, paclitaxel, topotecan.

\section{Introduction}

Fortunately in the United States, cervical cancer has become dramatically less common over the last five to seven decades ${ }^{1}$. Currently, 12,710 cases are discovered annually in the United States ${ }^{2}$. While the annual rate has dropped in the United States, in less developed countries more than 17 cases are found per 100,000 women $^{1}$. Cervical cancer has been closely related to low socio-economic status in developed countries $^{3}$. The economic burden to a country is significant and appears to be greater than $\$ 13,000 /$ patient diagnosed with a new case ${ }^{4}$. This number shifts significantly if patients are found with more advanced disease rather than early curable disease.

The Gynecologic Oncology Group (GOG) has published multiple papers examining the best chemotherapy regimen for treatment of women with advanced or recurrent cervical cancer. In 2004, Moore and colleagues published GOG 169 examining the survival differences in women with advanced, recurrent or persistent squamous cell carcinoma treated with cisplatin and those treated with cisplatin/paclitaxel $(\mathrm{CP})^{5}$. Although a two month increase in progression free survival was found, the overall 
survival was not different ( 8.8 months vs. 9.7 months) and there were increased complications in the $\mathrm{CP}$ arm. A year later, Long published GOG 179 examining a similar population treated with cisplatin versus cisplatin/topotecan $(\mathrm{CT})^{6}$. In Long's study, CT had a 2.9 month overall increase in survival over cisplatin (9.4 months vs. 6.5 months). This study was hailed as the first study to show an increased survival for combination therapy over cisplatin alone making CT the standard of care. No analysis of the cost such a small increase in survival would cause was performed. US healthcare related costs are expected to account for $25 \%$ of the gross domestic product by 20147 . With current governmental budgetary demands, this level of healthcare expenditure is not sustainable. Chemotherapeutic agents represent the most expensive drugs used by practitioners today, therefore it is prudent to examine its cost effectiveness.

Although there was a significant increase in survival, the addition of topotecan increases the cost of therapy and management of side effects. The objective of this study is to perform a cost effectiveness analysis of these two studies to determine which regimen is most cost effective. This analysis would hopefully allow a rational use of chemotherapy.

\section{Materials and Methods}

A decision analytic model was created to estimate the costs and outcomes of treating women with advanced, recurrent or persistent squamous cell carcinoma treated with cisplatin (arm 1) versus cisplatin with paclitaxel (CP) (arm 2) versus cisplatin with topotecan (CT) (arm 3) using a health system perspective (Figure 1) ${ }^{5,6}$. None of the authors had access to results from GOG 169 or 179 beyond what was published in the referenced articles. In the model

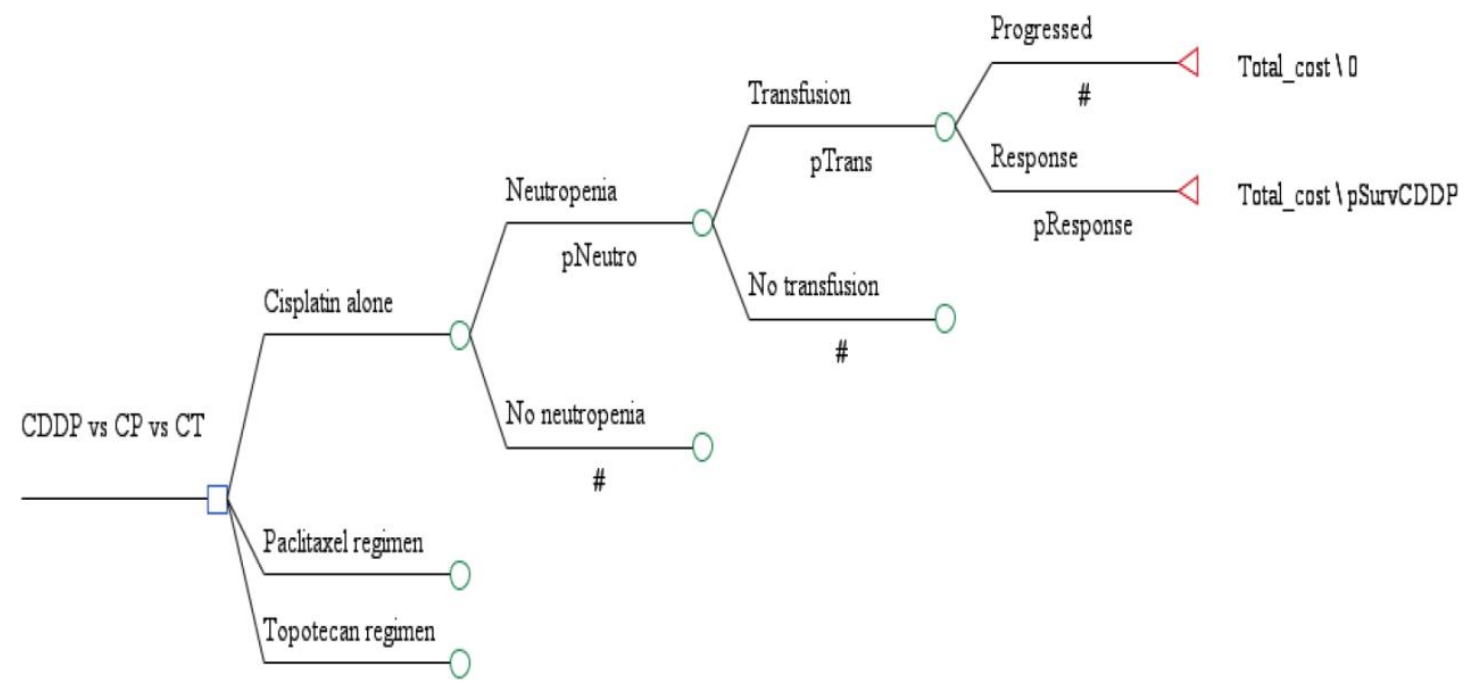

analysis, all patients were treated with 6 cycles of chemotherapy. An average patient was assumed to be a 65 year old with a body surface area of $2 \mathrm{~m}^{2}$ with a height of $165 \mathrm{~cm}$, weight of $100 \mathrm{~kg}$ and an ideal body weight of ideal body weight $57 \mathrm{~kg}$ and a creatinine of $0.8 \mathrm{mg} / \mathrm{dL}$. (Cockcroft \& Gault actual weight).

In arm 1, patients receive cisplatin $50 \mathrm{mg} / \mathrm{m}^{2} \mathrm{q}$ 21 days for 6 cycles. In arm 2 , all patients received cisplatin $50 \mathrm{mg} / \mathrm{m}^{2}$ and paclitaxel $135 \mathrm{mg} / \mathrm{m}^{2} \mathrm{q} 21$ days for 6 cycles. In arm 3, patients received 50 $\mathrm{mg} / \mathrm{m}^{2}$ on day $1 \mathrm{q} 21$ days for 6 cycles, and topotecan $0.75 \mathrm{mg} / \mathrm{m}^{2}$ on day 1,2,3 q 21 days for 6 cycles. Probabilities for complications, recurrence, and survival were derived from the results of the GOG 169 and GOG 179 randomized trials as detailed below ${ }^{5,6}$. We did not adjust complication, recurrence, or survival rates for age, and we assumed that experiencing a grade 3 or worse complication did not affect recurrence or survival rates. Utility values were used from literature ${ }^{8}$.

With additional agents added to cisplatin (paclitaxel or topotecan), the risk of grade 3 or greater complications increased but overall survival increased. These estimates were based on 2011 Medicare costs as well as published figures. The values used in the base case, as well as the ranges used for sensitivity analysis, are shown in Table 1.

Table I. Range in months over which survivals were varied.

\begin{tabular}{llll}
\hline Treatment & Base case & Low & High \\
\hline Cisplatin & 8.8 & 6.5 & 8.8 \\
CP & 9.7 & 4.9 & 14.6 \\
CT & 9.4 & 4.7 & 14.1 \\
\hline
\end{tabular}$$
\text { published in the referenced articles. In the model }
$$

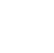


Because of the uncertainty surrounding all estimates, values were varied widely in sensitivity analysis to determine the effect on results. One-way sensitivity analysis was performed on all variables. Incremental cost-effectiveness was measured as cost per quality adjusted life year survival, calculated by dividing the difference in cost between the strategies by the difference in survival among the strategies. A willingness to pay threshold of $\$ 50,000$ per quality adjusted life year was chosen as an acceptable increase in cost.

\section{Results}

GOG 169 demonstrated no difference in overall survival with the CPPD arm having a survival of 8.8 months while the CP arm had an overall survival of 9.7 months. In contrast, GOG 179 showed that the CT arm was superior with an overall survival of 9.4 months versus the cisplatin arm's survival of only 6.5 months. These numbers made up the base case analysis. The difference in the cisplatin treated population's survival between 169 and 179 was accounted for in sensitivity analysis. Table 1 gives the survival and sensitivity data used in analysis.

Although the least effective drug in both GOG 169 and 179, cisplatin (arm1) was the most cost-effective. The combination $\mathrm{CP}$ did not significantly increase the survival over cisplatin alone per GOG 169 and gave an increased cost effectiveness ratio (ICER) of $\$ 13,654 / \mathrm{QALY}$. If the survival from GOG 179 for cisplatin was used instead, ICER would be $\$ 10,994 /$ QALY. Using the base case survival for cisplatin from GOG 169 and comparing it to the data from GOG 179, CT is dominated as compared to the other two regimens (Figure 2). As compared to cisplatin, CT does give increased overall survival but it does so at an ICER of $\$ 579,170 /$ QALY. Table 2 shows the one way sensitivity data varying the costs over the range specified in Table 1.

GOG 179 had a much lower survival for patients receiving cisplatin of 6.5 months compared to 8.8 months for patients on GOG 169. If this overall survival is used the ICER for CT decreases is $\$ 152,327 /$ QALY. Thus, even with the lower survival of GOG 179 with cisplatin it is still a more cost effective regimen compared to CT. To make this regimen cost effective with an ICER less than the WTP threshold of $\$ 50,000 /$ QALY the overall survival would have to increase to 24 months from the published 9.4 months. Even if the survival of CT were increased $50 \%$ to $>14$ months, the increased cost effectiveness ratio per quality adjusted life year gained is $\$ 66,400$.

\section{Cost-Effectiveness Analysis}

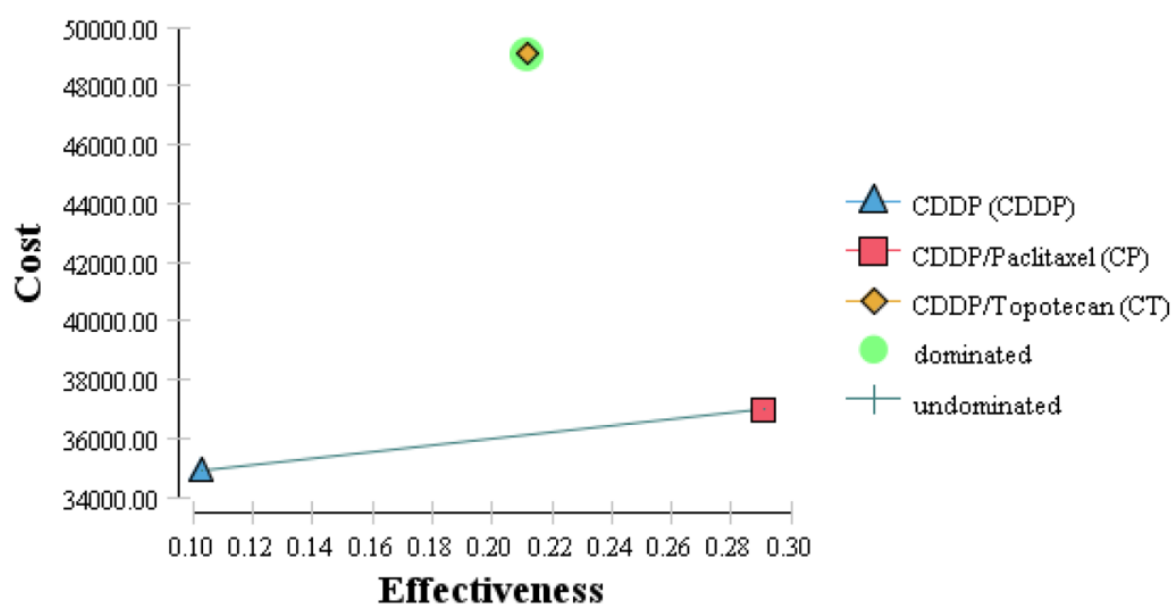

Figure 2. Cost effectiveness diagram with cost on the vertical access in dollars and relative effectiveness on the horizontal access. The shape legends are contained on the figure.

Table 2. Sensitivity analysis varying chemotherapy costs.

\begin{tabular}{llllll}
\hline Treatment & $\begin{array}{l}\text { Overall survival } \\
\text { months }\end{array}$ & $\begin{array}{l}\text { Chemotherapy costs alone / } \\
\text { patient }(\$)\end{array}$ & $\begin{array}{l}\text { Direct costs / patient } \\
(\$)\end{array}$ & $\begin{array}{l}\text { Direct and indirect costs / } \\
\text { patient }(\$)\end{array}$ & ICER / QALY \\
\hline Cisplatin & 6.5 & 95 & 2,077 & 34,908 & -- \\
CP & 9.7 & $489 \rightarrow 95$ & $2,737 \rightarrow 2,343$ & $36,978 \rightarrow 36,584$ & $10,994 \rightarrow 8,870$ \\
CT & 9.4 & $7,480 \rightarrow 95$ & $11,160 \rightarrow 3,775$ & $49,071 \rightarrow 41,686$ & $133,067 \rightarrow 62,246$ \\
\hline
\end{tabular}


Table 3. Sensitivity analysis varying chemotherapy costs.

\begin{tabular}{llll}
\hline Treatment & Overall survival months & Direct and indirect costs / patient $(\$)$ & ICER / QALY \\
\hline Cisplatin & 6.5 & 34,908 & -- \\
CP & $9.7 \rightarrow 14.6$ & 36,978 & $10,994 \rightarrow 6,150$ \\
CT & $9.4 \rightarrow 14.1$ & 49,071 & $133,067 \rightarrow 66,400$ \\
\hline
\end{tabular}

\section{Discussion}

Cisplatin chemotherapy is commonly used for the treatment of cervical cancer. This includes both as a radiation sensitizing agent and as a systemic treatment for advanced, persistent or recurrent cervical cancer 5 ,6, 9. When comparing GOG 169 and 179, the cisplatin doublets both had similar overall survivals of between 9 and 10 months while cisplatin had a large drop in survival from 8.8 months in GOG 169 to 6.5 months in GOG 179. It is not known whether this is just a random event or if it is demonstrating decreasing efficacy of cisplatin regimens because of increased use of the drug as a radiation sensitizer. This change needs to be followed up both clinically and from a cost effective analysis point of view to help design cost-effective future regimens. In Paton's analysis for the National Health Service in the United Kingdom, the authors had access to data which was not reported or specifically delineated in the referenced reports ${ }^{5,6}$.

The model analysis showed that although the survival in the cisplatin population is lower than in arm 2 or 3 , it is still the most cost effective medication currently studied. In GOG 169, CP increased overall survival from the cisplatin treated population's overall survival of 8.8 months to 9.7 months ${ }^{5}$. This difference was not found to be statistically significant. The ICER was $\$ 13,654 / Q A L Y$. If a survival of 6.5 months for cisplatin is used, the ICER is $\$ 10,994 /$ QALY. Although these increases are well below the standard acceptable WTP of $\$ 50,000 /$ QALY, there is no persuasive evidence to use $\mathrm{CP}$ as the new standard of care because GOG 169 did not show a significant increase in survival.

In GOG 179, the cisplatin population's overall survival dropped to 6.5 months. In comparison, the CT population's overall survival was 9.4 months. Although this was smaller than the survival found using CP in GOG 169, the 2.9 month increase was seen as a significant survival increase (P 0.015) ${ }^{6}$. The ICER compared to a cisplatin if a survival of 8.8 months is used is $\$ 194,578 /$ QALY and still $\$ 130,067 /$ QALY if a survival for the cisplatin population of 6.5 months is used.

The real point of cost analysis is to determine when a new paradigm should occur because a new regimen is both better (efficient) and not overly costly. Using the two studies (GOG 169 and 179) cisplatin in combination with the presented cost effective analysis data, cisplatin should still be considered the clinical standard of care. While $\mathrm{CP}$ had the longest survival, it was not statistically significant due to the better survival the cisplatin alone had in GOG 169 compared to 179. CT had a significantly longer survival than cisplatin, but it was not cost effective. The current economic environment requires us as physicians to realize that small increases in survival may not necessitate changes of standard of care if they are not economically feasible.

The National Institute for Health and Clinical Excellence (NICE) in the United Kingdom approved CT for use in a very select subset population ${ }^{10}$. This population was different than the general population and included only those without prior platinum exposure. That specific population was not studied specifically in the current article. NICE also had access to data from the articles that was not published ${ }^{10}$.

An alternative would be to change the cost of the medications. If the drug cost of for each 3 day cycle of topotecan were to drop from $\$ 1230$ to $\$ 0$, the overall cost would still be higher than cisplatin alone, and would still not fall under the WTP ratio of $\$ 50,000 /$ QALY $(\$ 62,246 /$ QALY). Even if the topotecan were free, it would be dominated by $\mathrm{CP}$ because CT has many more complications.

\section{Acknowledgements}

We thank Women's Oncology Research and Dialogue Foundation for support.

\section{Competing Interests}

The authors have declared that no competing interest exists.

\section{References}

1 Jemal A, Bray F, Center MM, Jerlay F, Ward E, Forman D. Global cancer statistics. CA Cancer J Clin 2011; 61: 69-90.

2 Siegel R, Ward E, Brawley O, Jemal A. Cancer statistics, 2011. CA Cancer J Clin 2011; 61: 212-36.

3 Robinson KM, Christensen KB, Ottesen B, Krasnik A. Socio-demographic factors, comorbidity and diagnostic delay among women diagnosed with cervical, endometrial or ovarian cancer. Eur J Cancer Care 2011; 20: 653-61.

4 Annemans L, Remy V, Lamure E, Spaepen E, Lamotte M, Muchada JP, Largeron N. Economic burden associated with the management of cer- 
vical cancer, cervical dysplasia and genital warts in Belgium. J Med Econ 2008; 11: 135-50.

5 Moore DH, Blessing JA, McQuellon RP, Thaler HT, Cella D, Benda J, Miller DS, Olt G, King S, Boggess JF, Rocereto TF. Phase III Study of Cisplatin With or Without Paclitaxel in Stage IVB, Recurrent, or Persistent Squamous Cell Carcinoma of the Cervix: A Gynecologic Oncology Group Study. J Clin Oncol 2004; 22: 3113-3119.

6 Long HJ, Bundy BN, Grendys EC, Benda JA,McMeekin DS, Sorosky JI, Miller DS,Eaton LA, Fiorica JV. Randomized Phase III Trial of Cisplatin With or Without Topotecan in Carcinoma of the Uterine Cervix: A Gynecologic Oncology Group Study. J Clin Oncol 2005; 23: 4626-4633.

7 Cohn DE, Kim KH, Resnick KE, O'Malley DM, Straughn JM, Jr. At what cost does a potential survival advantage of bevacizumab make sense for the primary treatment of ovarian cancer? A cost-effectiveness analysis. J Clin Oncol 2011; 29: 1247-51.

8 Versteegh MM, Leunis A, Uyl-de Groot CA, Stolk EA. Condition-specific preference-based measures: benefit or burden? Value Health 2012; 15: 504-13.

9 Peters WA, Liu PY, Barrett RJ, Stock RJ, Monk BJ, Berek JS, Souhami L, Grigsby P, Gordon W, Alberts DS. Concurrent Chemotherapy and Pelvic Radiation Therapy Compared With Pelvic Radiation Therapy Alone as Adjuvant Therapy After Radical Surgery in High-Risk Early-Stage Cancer of the Cervix. J Clin Oncol 2000; 18:1606-13.

10 Paton F, Paulden M, Saramago P, Manca A, Misso K, Palmer S, Eastwood A. Topotecan for the treatment of recurrent stage IB carcinoma of the cervix. Health Technol Assess 2010; 14(s1): 55-62. 